\title{
AD-HOC PRINCIPLES OF "MINIMUM ENERGY EXPENDITURE" AS COROLLARIES OF THE CONSTRUCTAL LAW THE CASES OF RIVER BASINS AND HUMAN VASCULAR SYSTEMS
}

\author{
A. Heitor Reis \\ Department of Physics and Institute of Earth Sciences (ICT), University of Évora, R. Romao Ramalho, 59, \\ 7002-554 Evora, Portugal \\ Email: ahr@uevora.pt
}

\begin{abstract}
In a recent paper [1] Reis showed that both the principles of extremum of entropy production rate, which are often used in the study of complex systems, are corollaries of the Constructal Law. In fact, both follow from the maximization of overall system conductivities, under appropriate constraints. In this way, the maximum rate of entropy production (MEP) occurs when all the forces in the system are kept constant. On the other hand, the minimum rate of entropy production $(\mathrm{mEP})$ occurs when all the currents that cross the system are kept constant.

In this paper it is shown how the so-called principle of "minimum energy expenditure" which is often used as the basis for explaining many morphologic features in biologic systems, and also in inanimate systems, is also a corollary of Bejan's Constructal Law [2].

Following the general proof some cases namely, the scaling laws of human vascular systems and river basins are discussed as illustrations from the side of life, and inanimate systems, respectively.
\end{abstract}

Keywords: Flow systems, Ad-hoc principles; Entropy production rate, Energy expenditure, Constructal Law.

\section{SYSTEM DYNAMICS, ENERGY EXPENDITURE, ENTROPY PRODUCTION, AND THE CONSTRUCTAL LAW}

Dynamics and evolution of complex systems are not easy to predict. This is why many studies of such systems generally invoke either principles of extremum of entropy production rate or extremum of "energy (power) expenditure" as the theoretical ground for explaining their dynamics.

The principle of Minimum Entropy Production rate (mEP) was first proposed by Prigogine $[3,4]$ as a rule governing open systems at nonequilibrium stationary states: "In the linear regime, the total entropy production in a system subject to flow of energy and matter, reaches a minimum value at the nonequilibrium stationary state" [4]. The justification of mEP presented by Prigogine still continues to be the subject of heated controversy.

The principle of Maximum Entropy Production rate (MEP) was proposed in 1956 by Ziman [5] in the form: "Consider all distributions of currents such that the intrinsic entropy production equals the extrinsic entropy production for the given set of forces. Then, of all current distributions satisfying this condition, the steady state distribution makes the entropy production a maximum".

The principle of "Minimum Energy Expenditure" has also been widely used. Classical examples from the side of living systems are the derivation of the scaling laws of branching in vascular systems by C. Murray [6-8], and from the inanimate side the studies on the tri-dimensional structure of river basins by Rodríguez-Iturbe et al. [9]. Interestingly, in the later work the authors also used the additional principle of "Equal Energy Expenditure".

Being taken as "principles" none of the above statements has been demonstrated, and therefore should be considered as "rational beliefs" whose validity and utility have to be inferred from the adequacy of their predictions to observational data. However, it is beyond doubt that so many "ad-hoc principles" generate some intellectual discomfort, and so it becomes clear that an effort is needed to unify the underlying theoretical framework for the study of complex systems.

In a recent paper [1] Reis showed how both mEP and MEP principles stem from Constructal Law as corollaries. The Constructal Law, which states: "For a finite-size system to persist in time (to live), it must evolve in such a way that it provides easier access to the imposed (global) currents that flow through it" [2], was translated into the mathematical form:

$$
d L_{i k}=0 ; d^{2} L_{i k}<0, \forall i, k
$$

where $L_{i k}$ is conductivity that couples force $\boldsymbol{F}_{k}$ with flow $\boldsymbol{J}_{i}$. The forces $\boldsymbol{F}_{k}=\nabla \Phi_{k}$ are the gradients of the potentials $\Phi=\{T, P, \mu, \ldots . \phi\}$. In fact, "easiest flow access" occurs when all conductivities $L_{i k}$ are at their maxima. The results may be summarized as follows [1]: 
- "In the case when all the forces driving the flows are kept constant the entropy production rate reaches the maximum value that is compatible with the existing constraints"

○ "In the case when all the flows are kept constant, the entropy production rate reaches the minimum value that is compatible with the existing constraints".

Therefore, the extrema of entropy production rate, which are often presented as principles governing the dynamics of certain systems, are in fact corollaries of the Constructal Law in certain specific situations. Moreover, in each situation the Constructal Law requires a specific flow organization as a fundamental condition for achieving either maximum, or minimum entropy production rate.

In what follows we show that the so-called "principles of extremum of energy expenditure" also follow as corollaries of the Constructal Law.

\section{THE "PRINCIPLES OF EXTREMUM OF ENERGY EXPENDITURE" AS COROLLARIES OF THE CONSTRUCTAL LAW}

Thought no unified formulation is found in the works that use these principles, the general idea is that many biologic and inanimate systems behave as if the whole "energy expenditure" required to sustain the internal processes was minimum. The derivation of the known scaling laws of dichotomous branching flow systems is such an example. For instance, Murray’s law for diameter scaling reads:

$$
D_{0}^{3}=D_{1}^{3}+D_{2}^{3}
$$

where the subscript 0 stands for parent vessel and subscripts 1 and 2 for daughter vessels. Murray [6,7] derived his law using biological considerations, but Sherman [8] showed that it could be derived by considering minimum total power expenditure. Total power was considered to be the power needed to drive the flow in the Poiseuille regime that scales with $D^{-4} L$ - where $L$ is vessel length - plus the "metabolic power" required to maintain the volume of blood and vessel tissue involved in the flow, which in a cylindrical vessel scales with $D^{2} L$. In fact, in Sherman's minimization, the consideration of a "metabolic power" in addition to power for driving the flow is mathematically equivalent to minimizing the power needed to drive the flow under total constant vessel volume (vessel volume $=\pi D^{2} L / 4$ ), as Bejan et al. [10] did by using the Constructal Law.

The scaling laws of branching pulsatile flows have been studied by Silva and Reis [11], who showed that they reduce to Murray's law at zero pulse frequency. Moreover, by using Constructal Law these authors were able to explain some physiological features such as the elongation of the ascending aorta with age [12], and also the different behaviour of the radial and carotid arteries with pulse frequency [13].

In their studies on the tri-dimensional structure of river basins Rodríguez-Iturbe et al. [9] found that known empirical scaling laws could be explained if the following theoretical framework was assumed: "(1) the principle of minimum energy expenditure in any link of the network, (2) the principle of equal energy expenditure per unit area of channel anywhere in the network, and (3) the principle of minimum total energy expenditure in the network as a whole". With respect to the scaling laws of river basins, studies published after the paper by Rodríguez-Iturbe et al. may be found in the literature that show that the Constructal Law alone was able to anticipate the empirical scaling laws $[14,15]$.

To understand the connection between the Constructal Law and ad-hoc "minimum energy expenditure" principles first let us note that "minimum energy expenditure" actually means "minimum flow exergy destruction". In fact the streams carry a flow exergy potential (per unit mass) that reads

$$
\psi=\left(h-h_{0}\right)+T_{0}\left(s-s_{0}\right)+v^{2} / 2+g z
$$

where $h$ stands for specific entalphy $\left(\mathrm{Jkg}^{-1}\right), T_{0}$ for ambient temperature $(\mathrm{K}), s$ for specific entropy $\left(\mathrm{JK}^{-1} \mathrm{~kg}^{-1}\right), v$ for velocity $\left(\mathrm{ms}^{-1}\right), g$ for acceleration due to gravity $\left(\mathrm{m}^{2} \mathrm{~s}^{-1}\right)$, and $z$ for height above a reference level $(\mathrm{m})$.

According to the Gouy-Stodola theorem: "In any open system, the rate of flow exergy lost for irreversibility $\dot{\Psi}=\dot{m} \psi$, (which is negative, and where $\dot{m}$ is mass flow rate) and the entropy generation rate $\dot{S}_{g e n}$ are related each another as $\dot{\Psi}=-T_{0} \dot{S}_{\text {gen }}$, where $T_{0}$ is the ambient temperature" (see for instance [16]).

Therefore by applying the Gouy-Stodola theorem to a flow tree with $N$ channels, one has

$$
\sum_{i=1}^{N} \dot{\Psi}_{i}=-T_{0} \sum_{i=1}^{N}\left(\dot{S}_{g e n}\right)_{i}
$$

In view of eq. (4), and the statements $A$ and $B$, it follows: A1 - When the entropy production rate reaches the minimum value, the rate of "energy expenditure" is also at the minimum;

B1 - When the entropy production rate reaches the maximum value, the rate of "energy expenditure" is also at the maximum.

Therefore it is clear that to each extremum of rate of "energy expenditure" corresponds the respective extremum of entropy generation rate. In this way, minimum "energy expenditure" is equivalent to minimum entropy production rate. As referred above, the so-called $\mathrm{mEP}$ principle is a corollary of the Constructal Law that is applicable when all currents are fixed, therefore the so-called principle of minimum "energy expenditure" is also a corollary of the Constructal Law.

In this context, the successful application of the principle of minimum "energy expenditure" to scaling of branching vessels (e.g. Murray's Law) in the case of biologic systems finds its justification in the fact that blood flow rate is set by the fixed needs of the organs and tissues supplied by the vascular tree. Hence, because the flow is fixed, the entropy generation rate is at its minimum, and same occurs with the rate of "energy expenditure".

Analogously, in the case of river basins, the global flow is fixed by the precipitation regime, hence the streams distribute in the basin in such a way that the entropy generation rate, and also the rate of "energy expenditure" are at their minima. This explains the successful application of the principle of minimum "energy expenditure" by Rodríguez-Iturbe et al. It is worth to say that Reis [14] and Bejan et al., [15] have anticipated the empirical scaling laws of river basins by using the Constructal Law alone, without needing to invoke any of the above mentioned ad-hoc principles. 
On the other side, some authors found that another ad-hoc principle, the "principle of maximum energy dissipation" was useful in describing patterns of water infiltration in cohesive soil with different populations of worm burrows for a range of rainfall scenarios [17]. They found that "flow in connected worm burrows allows a more efficient redistribution of water within the soil, which implies a more efficient dissipation of free energy/higher production of entropy". They explained that "this is because upslope run-off accumulates and infiltrates via the worm burrows into the dry soil in the lower part of the hillslope, which results in an overall more efficient dissipation of free energy". In fact, it is not surprising that the pattern corresponds to the maximum entropy production rate, given that water infiltrates into the soil under a fixed force (i.e. the gradient of water potential).

A classical application of the "maximum entropy production rate" was carried out by Paltridge [17-20] who proposed that the Earth's climate structure could be explained through the MEP principle. Also in this case, we can show that all the processes occurring in Earth that are powered by solar radiation occur in such a way that the net (whole) result is maximal entropy production rate.

For example, the processes involved in the transport of the excess heat absorbed at the equator (at temperature) $T_{H}$ to the poles, which are at a lower temperature $T_{L}$, develop to jointly produce maximum entropy per unit time. In fact, in this case, the global flow organizes itself in patterns that enable maximal "global heat conductivity" $[1,2]$, and because heat flows under a fixed force proportional to $\left(T_{H}-T_{L}\right)\left(T_{H} T_{L}\right)^{-1}$, maximal entropy production rate is expected to occur.

Many other cases in which the ad-hoc "principles of extremum of energy expenditure" are invoked as the theoretical basis for understanding system dynamics could be presented here. In all such cases it would be possible to show that they stem from a unique principle: the Constructal Law.

\section{CONCLUSIONS}

"Energy expenditure" in the sense of exergy destruction is related to entropy generation. Therefore, ad-hoc "principles of extremum of energy expenditure" are equivalent either to the "principle of minimum entropy production rate" - $\mathrm{mEP}$, or to the "principle of maximum entropy production rate" - MEP.

Both the mEP, and MEP principles are shown to be corollaries of the Constructal Law. Therefore, both the "principle of minimum energy expenditure", and the "principle of maximum energy expenditure", also are corollaries of the Constructal Law. In fact, taking together the statements A, A1, B, B1, from the Constructal Law it follows:

A2 - "In the case when all the forces driving the flows are kept constant the "energy expenditure" reaches the maximum value that is compatible with the existing constraints".

B2 - "In the case when all the flows are kept constant, the "energy expenditure" reaches the minimum value that is compatible with the existing constraints".

As the main conclusion: there is no need for using ad-hoc "principles of extremum of energy expenditure", because a unique principle - the Constructal Law -provides the theoretical basis for describing the dynamics of flow systems.

\section{ACKNOWLEDGMENT}

The author acknowledges the support provided by ICT under contract with FCT (the Portuguese National Science Foundation).

\section{REFERENCES}

1. Heitor Reis, A., "Use and validity of principles of extremum of entropy production in the study of complex systems," Annals of Physics 346, 22-27, 2014. DOI: 10.1016/j.aop.2014.03.013.

2. A. Bejan, Advanced Engineering Thermodynamics, 2nd ed. Wiley, New York, Ch. 13, 1997. ISBN: 978-0-47167763-5.

3. Prigogine, I., Étude Thermodynamique des Phenomenes Irreversibles, Desoer, Liege, Ch. V, 1947.

4. Kondepudi D., Prigogine, I., Modern Thermodynamics. From Heat Engines to Dissipative Structures, Wiley, Chichester, §17.2, 1998. DOI: 10.1002/9781118698723.

5. Ziman, J.M., "The general variational principle of transport theory," Canadian Journal of Physics 34, 1256-1273, 1956. DOI: 10.1139/p56-139.

6. Murray, C. D., "The physiological principle of minimum work: I. The vascular system and the cost of blood volume," Proc. of the National Academy of Sciences of the United States of America, 12 (3), 207-214, 1926. DOI: $10.1073 /$ pnas.12.3.207.

7. Murray, C.D., "The physiological principle of minimum work: II Oxygen exchange in capillaries," Proc. of the National Academy of Sciences of the United States of America, 12 (5), 299-304, 1926. DOI: 10.1073/pnas.12.5.299.

8. Sherman, T.F., "On connecting large vessels to small: The meaning of Murray's Law," The Journal of General Physiology, 78 (4), 431-453, 1981. DOI: 10.1085/jgp.78.4.431.

9. Rodríguez-Iturbe, I., Rinaldo, A., Rigon, R., Bras, R.L., Marani, A. and Ijjász-Vásquez, E., "Energy dissipation, runoff production, and the three-dimensional structure of river basins," Water Resources Research, 28 (4), 1095 1103, 1992. DOI: 10.1029/91WR03034.

10. Bejan, A., Rocha, L.A.O., and Lorente, S., "Thermodynamic optimization of geometry: T- and Yshaped constructs of fluid streams," Int. J. Thermal Sciences, 39, 949-960, 2000. DOI: 10.1016/S12900729(00)01176-5.

11. C. Silva, A.H. Reis, "Scaling relations of branching pulsatile flows," Int. J. of Thermal Sciences, 88, 77-83, 2014. DOI: $10.1016 /$ j.ijthermalsci.2014.09.009.

12. Silva, C. and Reis, A.H., "Structure and adaptation of arteries to pulsatile flow - The case of the ascending aorta," Medical Physics, 41, (6), 063701, 2014. DOI: $\underline{10.1118 / 1.4876379 .}$.

13. Silva, C. and Reis, A.H., "Heart rate, arterial distensibility, and optimal performance of the arterial tree," J. of Biomechanics, 47, (12), 2878-2882, 2014. DOI: 10.1016/j.jbiomech.2014.07.025.

14. Heitor Reis, A. "Constructal view of scaling laws of river basins," Geomorphology, 78, 201-206, 2006. DOI: 1016/j.geomorph.2006.01.015.

15. Bejan, A., Lorente, S., Miguel, A.F. and Reis, A.H., "Constructal theory of distribution of river sizes," $\$ 13$ 4, pp. 774-779, in Bejan, A., Advanced Engineering 
Thermodynamics, 3rd ed., Wiley, Hoboken, NJ, 2006. ISBN: 978-0-471-67763-5.

16. Bejan, A., Entropy Generation Minimization: The Method of Thermodynamic Optimization of Finite-Size Systems and Finite-Time Processes, Ch. 2, CRC Press, 1995. ISBN 9780849396519.

17. Zehe, E., Blume, T. and Blöschl, G., "The principle of 'maximum energy dissipation': a novel thermodynamic perspective on rapid water flow in connected soil structures," Phil. Trans. R. Soc. B, 365, 1377-1386, 2010. DOI: $10.1098 /$ rstb.2009.0308.
18. Paltridge, G.W., "Global dynamics and climate-A system of minimum entropy exchange," $Q . J . R$. Meteorol. Soc. 101, 475-484, 1975. DOI: 10.1002/qj.49710142906.

19. Paltridge, G.W., "The steady-state format of global climate," Q. J. R. Meteorol. Soc., 104, 927-945, 1978. DOI: 10.1002/qj.49710444206

20. Paltridge, G.W., "Climate and thermodynamic systems of maximum dissipation," Nature, 279, 630-631, 1979. DOI: $\underline{10.1038 / 279630 \mathrm{a} 0}$. 